\title{
Effects of long-term use of sodium-glucose co-transporter-2 inhibitors on plasma volume status in patients with type 2 diabetes mellitus: Sub-analysis of a prospective, observational study during the COVID-19 pandemic
}

\author{
Dimitrios Patoulias' ${ }^{1}$ Christodoulos Papadopoulos², loanna Zografou', Alexandra Katsimardou', \\ Konstantinos Imprialos ${ }^{1}$, Konstantinos Stavropoulos' ${ }^{1}$ Antigoni Tranidou', Asterios Karagiannis', \\ Michael Doumas ${ }^{1,3}$

\begin{abstract}
${ }^{1} 2^{\text {nd }}$ Propedeutic Department of Internal Medicine, Aristotle University of Thessaloniki, General Hospital "Hippokration", Thessaloniki, Greece ${ }^{2} 3^{\text {rd }}$ Department of Cardiology, Aristotle University of Thessaloniki, General Hospital “Hippokration”, Thessaloniki, Greece ${ }^{3}$ Veterans Affairs Medical Center, George Washington University, Washington, District of Columbia, United States of America
\end{abstract}

Correspondence to:

Dimitrios Patoulias, MD, $2^{\text {nd }}$ Propedeutic Department of Internal Medicine, General Hospital “Hippokration”, Konstantinoupoleos 49, 54642, Thessaloniki, Greece, phone: +30694 6900777 , e-mail: dipatoulias@gmail.com Copyright by the Author(s), 2022 DOI: 10.33963/KP.a2021.0160

\section{Received:}

October 12, 2021

Accepted:

November 14, 2021

Early publication date: November 16, 2021

\section{INTRODUCTION}

Type 2 diabetes mellitus (T2DM) constitutes a $21^{\text {st }}$-century epidemic [1]. Cardiovascular disease (CVD) represents the major cause of death among diabetic subjects. Sodium-glucose co-transporter-2 (SGLT-2) inhibitors have revolutionized the treatment of T2DM and concomitant CVD [3]. The drugs are also indicated in specific patient subgroups such as those suffering from heart failure with reduced ejection fraction or chronic kidney disease $[4,5]$. However, mechanisms underlying their beneficial effects remain largely unknown [6]. One of those proposed mechanisms is plasma volume (PV) contraction due to osmotic diuresis and natriuresis. Therefore, we sought to determine the effect of SGLT-2 inhibitors on plasma volume status (PVS), assessed non-invasively.

\section{METHODS}

This is a single-center, prospective, observational study conducted in Greece between January 2020 and July 2021. The study protocol was approved by the Ethics Committee of the School of Medicine, Aristotle University of Thessaloniki (protocol no. 4/17.7.2019). The study was performed in accordance with the principles outlined in the Declaration of Helsinki.

Subjects aged 18-75 years old, with a diagnosis of T2DM ( $\geq 12$ months), glycated hemoglobin $\left(\mathrm{HbA}_{1 \mathrm{c}}\right)$ values in the range of
6.5\%-10.0\%, stable antidiabetic and antihypertensive treatment over the last 6 months, and an indication for the initiation of an SGLT-2 inhibitor were eligible to participate.

All eligible patients provided written informed consent before enrollment. Enrolled participants were assigned either to therapy with dapagliflozin or empagliflozin. A follow-up visit was planned 6 months after the initiation of SGLT-2 inhibitor treatment. PVS was assessed with the equations described by Ling et al. [7].

\section{Statistical analysis}

Continuous variables are presented as mean (standard deviation [SD]), according to their normal distribution, while categorical variables are presented as relative frequencies (n [\%]). The Shapiro-Wilk test was used to test for normality. We performed hypothesis testing using a one-tailed paired t-test since all variables followed a normal distribution. Pearson correlation coefficient $(r)$ was used to assess the correlation of endpoint of interest (change in PVS, $\triangle \mathrm{PVS}$ ) with numerical variables of interest. Point-biserial correlation coefficient (rpb) was used to quantify the correlation between the continuous variable $\triangle$-PVS and the dichotomous variables. $P$-values $<0.05$ were considered statistically significant. $R$ software ( $R$ Foundation, Vienna, Austria) was utilized. 
Table 1. Participants' baseline characteristics

\begin{tabular}{lc}
\multicolumn{1}{c}{ Baseline characteristic } & Value \\
Enrolled subjects & 46 \\
Male sex & $29(63.04 \%)$ \\
Age, years & $62.89(8.53)$ \\
Bodyweight, kg & $90.04(17.4)$ \\
Body mass index, kg/m² & $31.25(5.8)$ \\
Glycated hemoglobin, \% & $7.48(1.51)$ \\
Type 2 diabetes mellitus duration, years & $9.72(6.37)$ \\
Arterial hypertension & $32(69.6 \%)$ \\
Dyslipidemia & $28(60.9 \%)$ \\
Cardiovascular disease & $26(56.5 \%)$ \\
Coronary artery disease & $14(30.4 \%)$ \\
Heart failure & $5(10.9 \%)$ \\
Atrial fibrillation & $1(2.2 \%)$ \\
Cerebrovascular disease & $7(15.2 \%)$ \\
Peripheral artery disease & $2(4.4 \%)$ \\
Chronic kidney disease & $3(5.5 \%)$ \\
Chronic obstructive pulmonary disease & $10(21.8 \%)$ \\
Obstructive sleep apnea & $7(15.2 \%)$ \\
\hline
\end{tabular}

Data are presented as mean (standard deviation [SD]) or absolute numbers unless otherwise stated

\section{RESULTS AND DISCUSSION}

Fifty-one subjects participated in the study. Two subjects refused to take part in follow-up visits, while 3 subjects discontinued treatment with SGLT-2 inhibitors due to mild adverse events. A total of 46 subjects with T2DM completed the study.

Their mean age was 62.89 (8.53) years, while the mean (SD) T2DM duration was 9.72 (6.37) years. Of enrolled participants, 29 were male, all Caucasian. Thirty patients were prescribed dapagliflozin and 16 empagliflozin. Despite the prespecified date of follow-up visit, COVID-19 pandemic regulations delayed follow-up visits, leading to a mean treatment duration of 9.98 (3.27) months.

The mean body mass index of enrolled subjects was $31.25(5.8) \mathrm{kg} / \mathrm{m}^{2}$, while their mean $\mathrm{HbA}_{1 c}$ was 7.48 (1.51) $\%$ at baseline (Table 1). A significant proportion of participants suffered from hypertension (69.5\%) and dyslipidemia (60.8\%), while $56.5 \%$ had pre-existing CVD. Regarding their antidiabetic treatment, $93.5 \%$ of patients received metformin, $28.3 \%$ received insulin, $32.6 \%$ were prescribed glucagon-like peptide-1 (GLP-1) receptor agonists, and $39.1 \%$ were administered dipeptidyl-peptidase-4 (DPP-4) inhibitors.

We demonstrated that long-term treatment with SGLT-2 inhibitors did not significantly affect PVS, since PVS changed from $-0.13(0.08) \%$ at baseline to $-0.15(0.09) \%$ at the end of follow-up period ( $\triangle \mathrm{PVS}=-0.02 \% ; P=0.99$ ). Notably, empagliflozin resulted in a non-significant change in PVS from $-0.16(0.06) \%$ to $-0.1575(0.1) \%(P=0.5)$. Dapagliflozin also led to a non-significant change in PVS from $-0.11(0.08) \%$ to $-0.14(0.08) \%(P=0.99)$. Interestingly, a history of pre-existing CVD did not significantly affect the observed results.
Pearson correlation coefficient analysis revealed that PVS correlated significantly with hematocrit $(r=-0.68$; $P<0.0001)$ and hemoglobin $(r=-0.66 ; P<0.0001)$. PVS did not correlate with other numerical variables.

We also documented a negative correlation between $\triangle \mathrm{PVS}$ and a history of coronary artery disease $(\mathrm{rpb}=-0.033$; $P=0.025)$ and a positive correlation with the prior use of DPP-4 inhibitors ( $r p b=0.3 ; P=0.042)$. No significant correlation was shown with a history of other major co-morbidities or prior antidiabetic or antihypertensive medication.

We demonstrated that long-term use of two different SGLT-2 inhibitors does not significantly affect PVS in patients with T2DM.

Dekkers et al. [8] demonstrated that dapagliflozin significantly decreased estimated PV at 24 weeks after initiation, showing a significant correlation with glycemic control, body weight, and creatinine clearance. Hoshika et al. [9] showed that empagliflozin produced a significant decrease in estimated PVS at 24 weeks in patients with T2DM and acute myocardial infarction. Similarly, Jensen et al. [10] observed that empagliflozin significantly decreased estimated PV at 12 weeks in patients with HF with reduced ejection fraction. Rapid attenuation of PV at 12 weeks has been shown with canagliflozin in patients with T2DM [11]. Finally, in the longest available study, long-term monotherapy with tofogliflozin in patients with T2DM produced a significant decrease in estimated PV 52 weeks post-initiation of treatment, however, that effect was rapidly ameliorated [12].

An interesting hypothesis has been suggested by Hallow et al. [13]. In healthy subjects, dapagliflozin led to a 3-fold decrease in interstitial fluid volume (IFV) compared to blood volume (BV), while the predicted decrease in IFV with bumetanide was only $80 \%$ of BV reduction [13]. A significant decrease in extracellular water volume with dapagliflozin has been shown in subjects with T2DM and established diabetic nephropathy [14]. Notably, Jensen et al. [10] showed that empagliflozin compared to placebo resulted in a significant decrease in estimated extracellular volume.

What we consider as major strengths of our study is the long follow-up period, the administration of two different SGLT-2 inhibitors, and running the study during the COVID-19 pandemic, which inevitably imposed significant limitations in patients' adherence to treatment, their physical status, and access to healthcare services. We acknowledge that our eligibility criteria were relatively broad, potentially missing the chance to address a significant effect of SGLT-2 inhibitors on PVS in specific patient populations.

To conclude, we demonstrated that long-term administration of two different SGLT-2 inhibitors in patients with T2DM does not affect PVS. Other mechanisms might be implicated with the beneficial effects of SGLT-2 inhibitors as a drug class. Osmotic diuresis, natriuresis, and blood pressure reduction, along with hematocrit and hemoglobin increase, decrease in serum uric acid, and improvement 
in albuminuria have been shown [6]. According to mechanistic studies, SGLT-2 inhibitors exert anti-inflammatory effects, enhance fat utilization and browning and suppress myocardial and renal oxidative stress. Improvement in myocardial energetics, reduction of cardiomyocyte apoptosis, and attenuation of sympathetic nervous system activity have also been proposed [6]. Some of these effects might also be valuable against COVID-19, which can prioritize the use of SGLT-2 inhibitors in subjects with T2DM during this hazardous pandemic [15].

Whether SGLT-2 inhibitors modify fluid volume regulation in the long term has to be clarified in future trials. In addition, it must be demonstrated if the hypothesis suggested by Hallow and colleagues [13], applies in clinical practice and in some patients' populations, such as those suffering from heart failure or diabetic nephropathy.

\section{Article information}

Study has been registered in the International Standard Registered Clinical/soCial sTudy Number (ISRCTN) registry (registration no. ISRCTN88851713).

\section{Conflict of interest: None declared.}

Open access: This article is available in open access under Creative Common Attribution-Non-Commercial-No Derivatives 4.0 International (CC BY-NC-ND 4.0) license, allowing to download articles and share them with others as long as they credit the authors and the publisher, but without permission to change them in any way or use them commercially. For commercial use, please contact the journal office at kardiologiapolska@ptkardio.pl.

\section{REFERENCES}

1. NCD Risk Factor Collaboration (NCD-RisC). Worldwide trends in diabetes since 1980: a pooled analysis of 751 population-based studies with 4.4 million participants. Lancet. 2016; 387(10027): 1513-1530, doi: 10.1016/S0140-6736(16)00618-8, indexed in Pubmed: 27061677.

2. Roth GA, Forouzanfar MH, Moran AE, et al. Demographic and epidemiologic drivers of global cardiovascular mortality. N Engl J Med. 2015; 372(14): 1333-1341, doi: 10.1056/NEJMoa1406656, indexed in Pubmed: 25830423.

3. Buse JB, Wexler DJ, Tsapas A, et al. 2019 Update to: Management of Hyperglycemia in Type 2 Diabetes, 2018. A Consensus Report by the American Diabetes Association (ADA) and the European Association for the Study of Diabetes (EASD). Diabetes Care. 2020; 43(2): 487-493, doi: 10.2337/dc20-er07, indexed in Pubmed: 32444453.
4. Sarafidis P, Ortiz A, Ferro CJ, et al. Sodium-glucose co-transporter-2 inhibitors for patients with diabetic and nondiabetic chronic kidney disease: a new era has already begun. J Hypertens. 2021; 39(6): 1090-1097, doi: 10.1097/HJH.0000000000002776, indexed in Pubmed: 33443971.

5. Packer M, Butler J, Filippatos G, et al. SGLT2 inhibitors in patients with heart failure with reduced ejection fraction: a meta-analysis of the EMPEROR-Reduced and DAPA-HF trials. Lancet. 2020; 396(10254): 819-829, doi: 10.1016/S0140-6736(20)31824-9, indexed in Pubmed: 32877652.

6. Cowie MR, Fisher M. SGLT2 inhibitors: mechanisms of cardiovascular benefit beyond glycaemic control. Nat Rev Cardiol. 2020; 17(12): 761-772, doi: 10.1038/s41569-020-0406-8, indexed in Pubmed: 32665641.

7. Ling HZ, Flint J, Damgaard $M$, et al. Calculated plasma volume status and prognosis in chronic heart failure. Eur J Heart Fail. 2015; 17(1): 35-43, doi: 10.1002/ejhf.193, indexed in Pubmed: 25469484.

8. Dekkers CCJ, Sjöström CD, Greasley PJ, et al. Effects of the sodium-glucose co-transporter-2 inhibitor dapagliflozin on estimated plasma volume in patients with type 2 diabetes. Diabetes Obes Metab. 2019; 21(12): 2667-2673, doi: 10.1111/dom.13855, indexed in Pubmed: 31407856.

9. Hoshika Yu, Kubota Y, Mozawa K, et al. Effect of empagliflozin versus placebo on plasma volume status in patients with acute myocardial infarction and type 2 diabetes mellitus. Diabetes Ther. 2021; 12 (8): 2241-2248, doi: 10.1007/s13300-021-01103-0, indexed in Pubmed: 34236577.

10. Jensen J, Omar M, Kistorp C, et al. Effects of empagliflozin on estimated extracellular volume, estimated plasma volume, and measured glomerular filtration rate in patients with heart failure (Empire HF Renal): a prespecified substudy of a double-blind, randomised, placebo-controlled trial. Lancet Diabetes Endocrinol. 2021; 9(2): 106-116, doi: 10.1016/S22138587(20)30382-X, indexed in Pubmed: 33357505.

11. Sha S, Polidori $D$, Heise $T$, et al. Effect of the sodium glucose co-transporter 2 inhibitor canagliflozin on plasma volume in patients with type 2 diabetes mellitus. Diabetes Obes Metab. 2014; 16(11): 1087-1095, doi: 10.1111/dom.12322, indexed in Pubmed: 24939043.

12. Matsubayashi Y, Yoshida A, Suganami H, et al. Association of estimated plasma volume and weight loss after long-term administration and subsequent discontinuation of the sodium-glucose cotransporter-2 inhibitor tofogliflozin. Diabetes Obes Metab. 2021; 23(7): 1660-1665, doi: 10.1111/dom.14387, indexed in Pubmed: 33769665.

13. Hallow KM, Helmlinger G, Greasley PJ, et al. Why do SGLT2 inhibitors reduce heart failure hospitalization? A differential volume regulation hypothesis. Diabetes Obes Metab. 2018; 20(3): 479-487, doi: 10.1111/dom.13126, indexed in Pubmed: 29024278.

14. Ohara K, Masuda T, Murakami T, et al. Effects of the sodium-glucose cotransporter 2 inhibitor dapagliflozin on fluid distribution: A comparison study with furosemide and tolvaptan. Nephrology (Carlton). 2019; 24(9): 904-911, doi: 10.1111/nep.13552, indexed in Pubmed: 30578654.

15. Patoulias D, Papadopoulos C, Kassimis G, et al. Cardiovascular drug therapy and surrogate COVID-19 outcomes: which is the impact of the "miraculous" sodium-glucose co-transporter-2 inhibitors? Kardiol Pol. 2021; 79(9): 1048-1049, doi: 10.33963/KP.a2021.0067, indexed in Pubmed: 34292565. 